ORIGINAL ARTICLE

\title{
Kikuchi's disease (KD); mimicking malignant lymphoma: A rare cause of cervical lymphadenopathy.
}

Ihsan Ullah', Muhammad Khizar Hayat ${ }^{2}$, Sanaullah Khan ${ }^{3}$, Nazish Khan ${ }^{4}$, Chaand Bibi ${ }^{5}$, Maham Rehman ${ }^{6}$

Article Citation: Ihsan Ullah, Hayat MK, Khan S, Khan N, Bibi C, Rehman M. Kikuchi's disease (KD); mimicking malignant lymphoma: A rare cause of cervical lymphadenopathy. Professional Med J 2022; 29(3):323-327. https://doi.org/10.29309/TPMJ/2022.29.03.6627

\begin{abstract}
Objective: The aim of this study is to present six cases of cervical lymphadenopathy, diagnosed as Kikuchi's disease and raise the index of suspicion for this disease. Study Design: Case Series. Setting: Histopathology and Otolaryngology/Head \& Neck Surgery Department at Rehman Medical Institute (RMI) Peshawar. Period: July 2016 to March 2021. Material \& Methods: Records of patients for cases diagnosed as "histiocytic necrotising lymphadenitis" were retrieved, their corresponding patient files accessed from the archive, and case notes analyzed. Results: In this series, a total of six cases of Kikuchi's disease are presented. A female predominance is evident from the present data (2:1 female to male ratio). All patients presented with palpable lymph nodes in the neck, predominantly on the left (66.7\%). Majority (66.7\%) presented with neuralgic pain (on/off). Only one patient did not show weight loss and night sweating, the remaining had all the symptoms. All of the patients recovered spontaneously within 6 months (range 2 to 6 months) with symptomatic treatment for neuralgic pain. Conclusion: Kikuchi's disease should be kept in the differential diagnosis in young females with persistent neck masses, palpable lymph nodes and suspicious fine needle aspiration cytology (FNAC).
\end{abstract}

Key words: Biopsy, Histiocytic Necrotizing Lymphadenitis, Kikuchi Disease, Lymph Nodes, Otolaryngology.

\section{INTRODUCTION}

Histiocytic necrotizing lymphadenitis or Kikuchi's disease (KD) was first described in 1972 by $\mathrm{Dr}$ Masahiro Kikuchi and Fujimoto in the Japanese literature..$^{1,2}$ It is an increasingly recognised, selflimiting cervical lymphadenitis of unknown origin predominantly affecting young women under the age of 30 , with men having a wider range of presentation ages. $^{3}$

The classical presentation is a unilateral painful posterior neck lymphadenopathy, fever, in a young adult, usually female. Less commonly it also involves axillary and abdominal lymph nodes. Parotid gland and spleen enlargement with skin rashes is also reported. ${ }^{4}$ The exact cause of KD is still not known but autoimmune and infections are considered as a possible aetiology. The Gold Standard for the diagnosis of $K D$ is lymph node biopsy with histological and microscopic confirmation of paracortical necrosis and histiocytic infiltration. The serological test like antinuclear antibodies (ANAs), anti-double strand antibodies, rheumatoid factor are usually negative in patients with $K D$. The association between KD and systemic lupus erythematosus (SLE) is mentioned in certain literature. ${ }^{5}$ Lymphadenopathy due to SLE is clinically and histologically similar to KD but the manifestations are different as reported by $\mathrm{Chua} \mathrm{SH}$, et.al. ${ }^{6}$

Any patient presenting with persistent neck lymph nodes, especially if young females, with high temperature, high or normal Erythrocyte sedimentation rate (ESR)/C-reactive Protein (CRP) and a suspicious fine needle aspiration cytology (FNAC) report should undergo excision biopsy to rule out KD.

The aim of this study is to present six cases of cervical lymphadenopathy, diagnosed as Kikuchi's disease and raise the index of suspicion
1. MBBS, FRCS (ORL), Associate Professor ENT, Rehman Medical Institute (RMI) Peshawar. 2. MBBS, Medical Officer, Rehman Medical Institute (RMI) Peshawar.

3. MBBS, House Officer, Naseer Teaching Hospital Peshawar.

4. MBBS, House Officer, Naseer Teaching Hospital Peshawar.

5. MBBS, House Officer, Rehman Medical Institute (RMI) Peshawar.

6. MBBS, House Officer, Rehman Medical Institute (RMI) Peshawar.

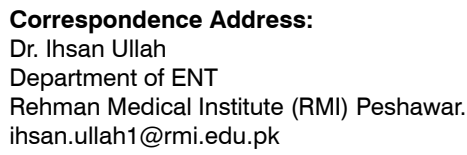

Correspondence Address:

Dr. Ihsan Ullah

Department of ENT

Rehman Medical Institute (RMI) Peshawar. ihsan.ullah1@rmi.edu.pk

Article received on: $08 / 06 / 2021$ Accepted for publication: 
for this disease.

\section{MATERIAL \& METHODS}

This study was conducted at the Histopathology Department and Department of Otolaryngology/ Head \& Neck Surgery of a Tertiary Care Hospital of Peshawar. A manual search through the histopathology department archives from July 2016 till March 2021 for cases diagnosed as "histiocytic necrotising lymphadenitis" was conducted which resulted in six cases. Pathologist reviewed the slides again and diagnosis were reconfirmed. Patient records were retrieved, case notes analysed, and relevant data collected.

\section{Illustrative Case}

A 19-year-old female, presented to ENT outpatient department with right sided palpable neck lymph nodes, on/off pain in the right side of neck, night sweats, and weight loss for the past 5 months. Prior to this, she was otherwise in good health apart from hospitalization for typhoid fever two years ago. There was no family history for any disease. Clinical exam shows a firm, non-tender, palpable lymph nodes on the right neck, mobile, no erythema, edema or brawny induration seen.

The patient had a routine complete blood count (Hemoglobin (Hb): 11.5gm/dcl White Blood Cell Count (WBC): $8.9 / \mathrm{mcl}$ Platelets: $335,000 / \mathrm{mm}^{3}$, CRP $(2.5 \mathrm{mg} / \mathrm{L}$ raised) and ANCA (Negative). A chest X-ray was also done which showed cervical lymphadenopathy. Ultrasound of the neck showed multiple lymph nodes, up to $3 \mathrm{~cm}$ in size and loss of fatty hilum. Fine Needle Aspiration Cytology (FNAC) was done for her which yielded, high cellularity with polymorphous population of lymphocytes. An excision biopsy was advised and subsequently performed. The sections showed extensive area of necrosis with multiple macrophages. No granuloma or malignancy was noted. The patient was labelled as a case of histiocytic necrotising lymphadenitis or Kikuchi's disease.

The patient was counselled about the disease, acetaminophen for pain management was advised and the patient was discharged with follow-up in 1 month. Symptomatic improvement was seen in the patient on the next visit and further follow-up after another month was advised. Spontaneous resolutions of the lymph nodes and symptoms occurred within 6 months of initial presentation. The recovery was uneventful.

\section{RESULTS}

In this series, a total of six cases of Kikuchi's disease are presented. A female predominance is evident from the present data (2:1 female to male ratio). Four of the patients were below 20 years of age while the remaining two were 30 and 39 years respectively. All patients presented with palpable lymph nodes in the neck, predominantly on the left (66.7\%). Majority (66.7\%) presented with neuralgic pain (on/off). Only one patient did not show weight loss and night sweating, the remaining had all the symptoms. Most of the patients had these symptoms for 3 to 6 months. Table-I shows the presenting complains.

\begin{tabular}{|l|l|l|}
\hline \multicolumn{2}{|c|}{ Symptom } & N (\%) \\
\hline \multirow{2}{*}{$\begin{array}{l}\text { Palpable Lymph } \\
\text { Nodes }\end{array}$} & Right & $1(16.7)$ \\
\cline { 2 - 3 } & Left & $4(66.7)$ \\
\cline { 2 - 3 } & Bilateral & $1(16.7)$ \\
\hline Neuralgic Pain (on/off) in neck & $4(66.7)$ \\
\hline Night Sweats & $5(83.3)$ \\
\hline Weight Loss & $5(83.3)$ \\
\hline \multicolumn{2}{|c|}{ Table-I. Presenting complains. } \\
\hline
\end{tabular}

None of the patients reported of fever. Ultrasound examination was done for all patients, majority of the findings were multiple lymph nodes ranging between $2 \mathrm{~cm}$ and $3.5 \mathrm{~cm}$.

Fine needle aspiration cytology (FNAC) was performed on all patients which revealed high cellularity with polymorphous population of lymphocytes in four cases while two cases showed histiocytic aggregates with intermediate sized lymphoid cells. Excision biopsy was recommended on all six samples. Five excision biopsy samples yielded paracortical necrosis with starry sky pattern and foamy histiocytes (Figure-1) with one showing extensive area of necrosis and multiple macrophages (Figure-2). 


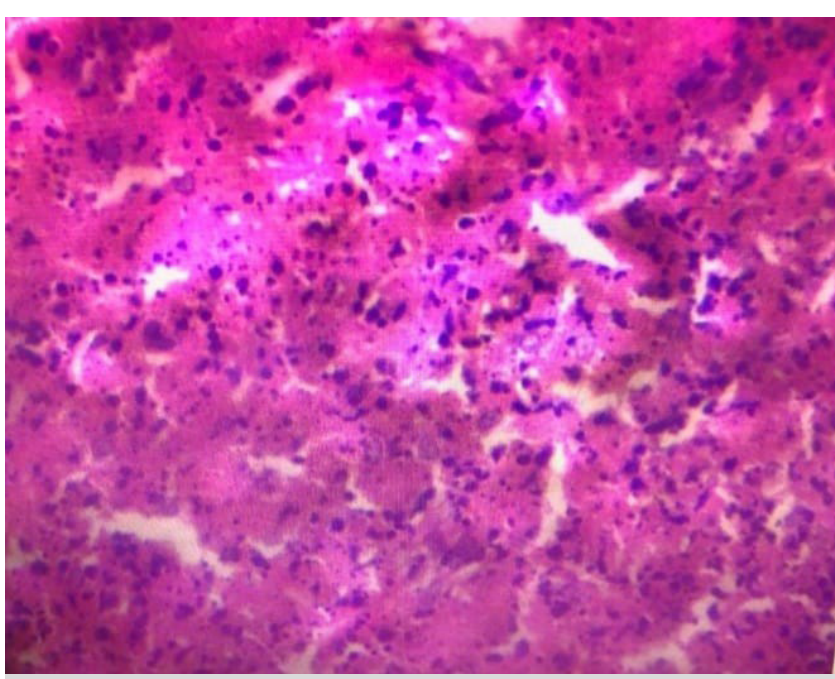

Figure-1. Paracortical necrosis with starry sky pattern, foamy histiocytes.

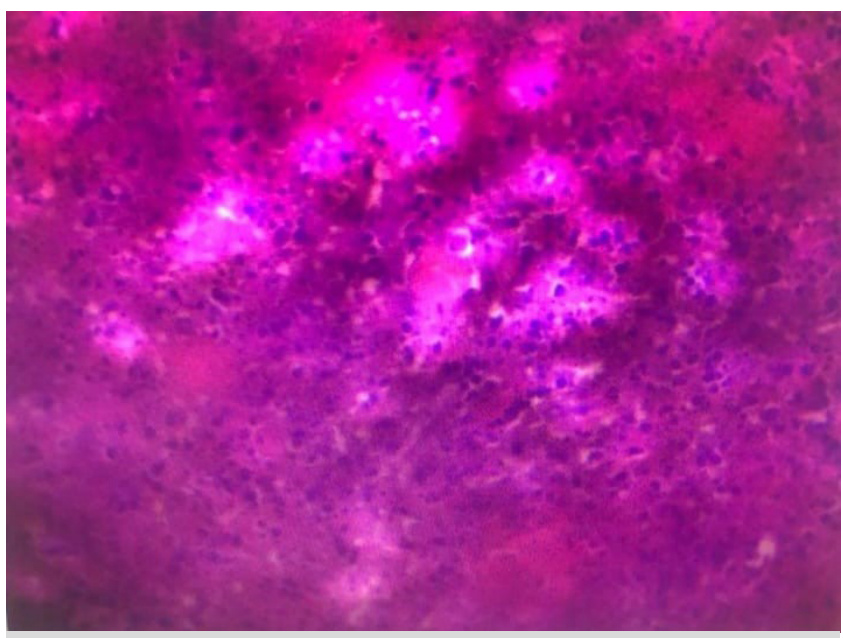

Figure-2. Histopathologic section of Kikuchi's disease.

Blood investigations did not show any characteristic findings with all samples having a normal complete blood count (CBC) and negative ANCA. Half the cases had raised C-reactive protein (CRP).

All six patients had their X-rays done which showed prominent lymph nodes. One of the patient's X-rays is shown in Figure-3.

Most of the patients did not have any significant past medical or surgical history however, one patient was asthmatic. All of the patients recovered spontaneously within 6 months (range 2 to 6 months) with symptomatic treatment for neuralgic pain.

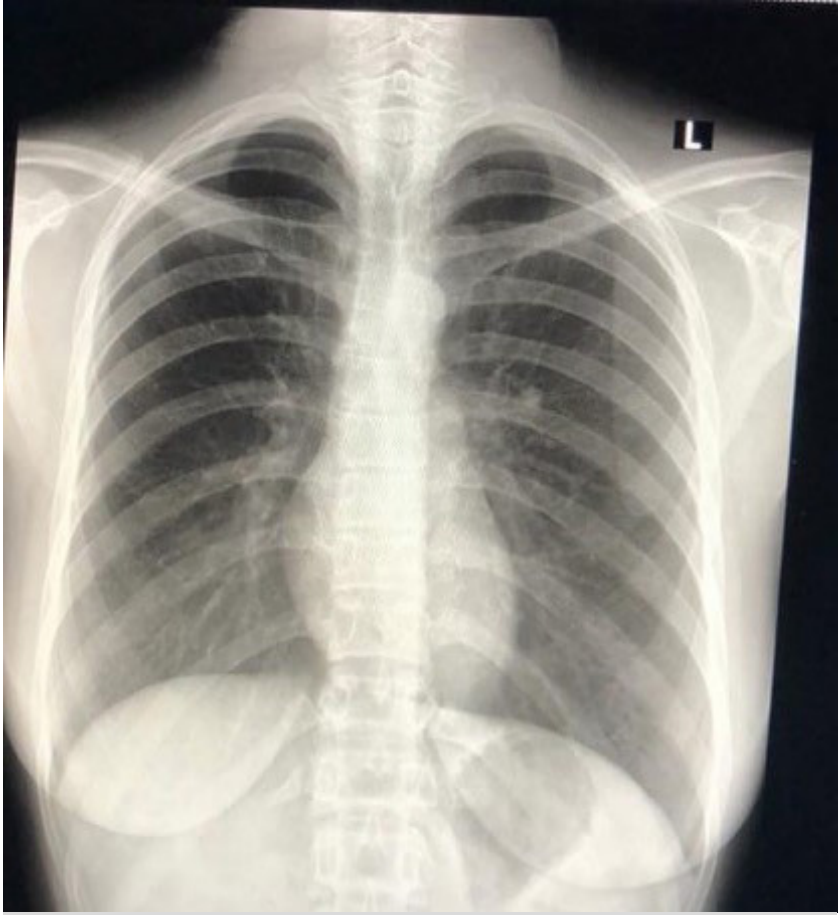

Figure-3. Cervical lymphadenopathy.

\section{DISCUSSION}

Kikuchi and Fujimoto in Japan first described Kikuchi's disease in 1972. The exact cause of Kikuchi's disease is still unknown but occurs sporadically in people with no family history of this condition, however, it is relatively benign and does not result in loss of life. ${ }^{7}$

A viral or post viral reaction has been proposed as its aetiology and several other infectious agents like Epstein Barr virus, Herpes simplex virus 2, cytomegalovirus (CMV), and Varicella Zoster virus have all been suggested but not confirmed till date. Other aetiological agents including HIV virus, Yersinia enterocolitica and silicone breast implants have also been proposed as possible aetiological agents. ${ }^{8,9}$

Most patients with this disease have been reported in Asian origin. A case from India has also been reported. ${ }^{10}$ Only a few individual case reports of KD are published in Pakistan. ${ }^{9,11,12}$

No diagnostic laboratory tests are available for the diagnosis of Kikuchi's disease, FNAC has been reported to be diagnostic by Hseuh EJ, 
et al. in 1992 however, the disease has been misdiagnosed as lymphoma due to it and some patients have even received chemotherapy. ${ }^{13,14}$

The diagnosis is usually confirmed by a lymph node biopsy, which shows the typical pathology of the disease and differentiates it from lymphoma, systemic lupus erythematosis, and other infectious lymphadenopathies. Hence, a clinician needs to keep this disease as a premium differential while diagnosis cases of neck masses/ swelling. We based our diagnosis on excision biopsy of the lymph nodes, with histological features of eosinophilic necrosis, monocytes, T-immunoblast and absent neutrophils.

Clinically, the disease presents with multiple lymphadenopathies, typically in head and neck region, although it may be generalised. The affected lymph nodes are generally multiple, firm, and mildly tender and specifically one being unilateral cervical lymphadenopathy. ${ }^{15}$ Splenomegaly may be present. Other symptoms like fever, nausea, vomiting, headache, fatigue, night sweats and weight loss may be present. ${ }^{16}$ Cutaneous skin manifestations have also been reported with cases of $\mathrm{KD} .{ }^{17}$ There was no any cutaneous lesion in all our cases.

The disease is self-limiting and benign in nature, but multiple recurrences have been reported in the same patient. ${ }^{18} \mathrm{~A}$ few fatalities like sudden heart failure and lupus like syndromes have occurred.

Some clinicians, because of its recurring nature and association with systemic lupus erythematosus recommend long-term follow up. Because of unknown its aetiology, symptomatic treatment with non-steroidal anti-inflammatory drugs is usually done. The use of steroids or other immunosuppressant drugs in selected cases to prevent fatal outcome have also been recommended. ${ }^{19}$ In other reports, oral prednisolone is shown to limit prolonged fever and other irritating symptoms lasting for more than two weeks. In the previous literature use of steroid was limited to Kikuchi's associated brachial plexus neuritis. ${ }^{20}$ FNAC has high sensitivity to identify metastatic carcinoma and melanoma in a lymph nodes. In lymphoid tumour the false negative rate is very high but FNAC with excision biopsy of lymph nodes increase the diagnostic accuracy to around $85-90 \% .^{21}$

\section{CONCLUSION}

Patients with cervical lymphadenopathy and suggested malignant lymphoma on cytology may be misdiagnosed as lymphoma and hence should have excision biopsy to confirm the diagnosis. Symptomatic treatment should be the mainstay for this benign disease, steroids and other immunosuppressant therapy should be reserved for more prolonged and aggressive forms of Kikuchi's disease.

\section{Copyright@ 25 Aug, 2021.}

\section{REFERENCES}

1. Bosch X, Guilabert A. Kikuchi-Fujimoto disease. Orphanet J Rare Dis. 2006; 1:18. doi: 10.1186/17501172-1-18.

2. Primrose WJ, Napier SS, Primrose AJ. KikuchiFugimoto disease (Cervical Subacute Necrotising Lymphadenitis): An important benign disease often masquerading as Iymphoma. Ulster Med J. 2009; 78(2):134-6. PMID: 19568451.

3. Bhat NA, Hock YL, Turner NO, Gupta ARD. Kikuchi's disease of the neck (histocytic necrotizing lymphadenitis). J Laryngol Otol. 1998; 112(9):898-900. doi: $10.1017 / \mathrm{s} 0022215100142021$.

4. Sato $Y$, Kuno $H$, Oizumi $K$. Histiocytic necrotizing lymphadenitis (Kikuchi's Disease) with aseptic meningitis. J Neurol Sci. 1999; 163(2):187-91. doi: 10.1016/s0022-510x(99)00037-4.

5. Hutchinson CB, Wang E. Kikuchi's -Fujimoto disease. Arch Pathol Lab Med. 2010; 134(2):289-93. doi: 10.5858/134.2.289.

6. Chua SH, Gium YC, Sim CS. Systemic lupus erythematosis with berythema multiform-like lesions and histiocytic necrotizing lymphadenitis- A case report. Ann Acad Med Singap. 1996; 25(4):599-601. PMID: 8893939.

7. Barbat B, Jhaj R, Khurram D. Fatality in KikuchiFujimoto disease: A rare phenomenon. World $\mathrm{J}$ Clin Cases 2017; 5(2):35-39. doi:10.12998/wjcc.v5.i2.35. 
8. Pileri AS, Pileri A, Yasukawa K, Kuo TS, Sullivan K. The Karma of Kiluchi's disease. Clin Immunol. 2005; 114:27-9. doi:10.1016/j.clim.2004.03.022.

9. Sultan S, Mushtaq H, Manan A. Kikuchi-Fujimoto diseasae: An important differential of tuberculous lympha- denitis. J Coll Physicians Surg Pak. 2020; 30(9):987-988. doi:10.29271/jcpsp.2020.09.987.

10. Rakesh P, Alex RG, Varghese GM, Mathew P, David T, Manipadam MT, et al. Kikuchi-Fujimoto disease: Clinical and laboratory characteristics and outcome. J Glob Infect Dis 2014; 6:147-50.

11. Mahmood S, Saif T, Khurram M, Khan MU. KikuchiFujimoto disease complicated by tuberculosis. Journal of Rawalpindi Medical College (JRMC). 2015; 19(1):102-104.

12. Rahman MSU, Muktadir G, Ahmed SAM. Kikuchi's disease: A case report. Faridpur Med. Coll. J. 2013; 8(2):96-98.

13. Hsueh EJ, Ko WS, Hwang WS, Yam LT. Fine needle aspiration of histiocytic necrotizing lymphadenitis (Kikuchi's disease). Diagn Cytopathol. 1993; 9(4):44852. doi: 10.1002/dc.2840090415.

14. Dorfman RF, Berry GI. Kikuchi's histiocytic necrotizing lymphadenitis: An analysis of 108 cases with emphasis on differential diagnosis. Semin Diagn Patho. 1988; 5(4):329-45.
15. Deaver D, Horna P, Cualing H, Sokol L. Pathogenesis, diagnosis and management of Kikichi-Fujimoto disease. Cancer control. 2014; 21(4):313-21. doi: 10.1177/107327481402100407.

16. Tati S, Kumar PBR, Yugandhar E, Praneeth K, Ramjo KVV. Kikuchi-Fujimoto disease: A rare case representation and review of literature. Journal of Evolution of Medical and Dental Sciences. 2015; 4(13):2214-17. doi: 10.14260/jemds/2015/318.

17. Jang JM, Woo CH, Choi JW, Song DJ, Yoo Y, Lee KC, et.al. A case of Kikuchi's disease with skin involvement. Korean Jouranl of Pediatrics. 2006; 49(1):103-6.

18. Smith KG, Becker GJ, Busmanis I. Recurrent Kikuchi's disease. Lancet. 1992; 340(8811):124. doi:10.1016/0140-6736(92)90454-b.

19. Shih-Hua L, Wang-sheng K, Herng-sheng L, Shyou $H$. Kikuchi's disease associated with lupus-like syndrome-A fatal case. JRheumatol. 1992:19(12):19956.

20. Sugiyama A, Araki E, Arakawa K, Kikuchi H, Iwaki T, Yamada $T$, et al. A case of subacute necrotizing lymphadenitis complicated with brachial plexus neuritis. Rinsho Shinkeigaku. 1998; 38(10-11):941944. PMID: 10203979.

21. Sneigge N. Diagnosis of lymphoma and reactive lymphoid hyperplasia by immunocytochemical analysis of fine needle aspiration biopsy. Diagn Cytopathol 1990; 6(1):39-43. doi: 10.1002/ dc. 2840060109 .

\begin{tabular}{|c|c|c|c|}
\hline \multicolumn{4}{|c|}{ AUTHORSHIP AND CONTRIBUTION DECLARATION } \\
\hline No. & Author(s) Full Name & Contribution to the paper & Author(s) Signature \\
\hline 2 & M. Khizar Hayat & $\begin{array}{l}\text { Conceived and designed did data } \\
\text { collection/correction \& final approval } \\
\text { of the manuscript. } \\
\text { Editing review \& final approval of the } \\
\text { manuscript. }\end{array}$ & \\
\hline 3 & Sanaullah Khan & Data collection \& manuscript writing. & \\
\hline 4 & Nazish Khan & Data collection \& manuscript writing. & \\
\hline 5 & Chaand Bibi & Data analysis \& Results generation. & chaond \\
\hline 6 & Maham Rehman & Data analysis \& Results generation. & Mahor \\
\hline
\end{tabular}

